\title{
Renal involvement in leprosy: evaluation of patients in Turkey
}

\author{
Savas Ozturk ${ }^{1}$, Tulin Ozturk², Ilkay Can ${ }^{1}$ \\ ${ }^{1}$ Department of Dermatology, Medical Faculty, Balikesir University, Balikesir, Turkey \\ 2Department of Radiology, Ataturk State Hospital, Balikesir, Turkey
}

Adv Dermatol Allergol 2017; XXXIV (3): 240-244
DOI: https://doi.org/10.5114/ada.2017.67846

\begin{abstract}
Introduction: Renal involvement in leprosy has previously been described in the literature and can include amyloidosis, glomerulonephritis, nephrosclerosis, tubulointerstitial nephritis, and granulomas.

Aim: To evaluate renal involvement in Turkish patients with leprosy.

Material and methods: In total, 32 patients with lepromatous leprosy but without any co-morbidities and 35 healthy control subjects were evaluated for renal involvement at the Elazig Training and Research Hospital in Turkey. The laboratory tests and radiological results concerning renal function were taken from both the patients' medical records and from current examinations.

Results: The levels of creatinine, urea, and leukocyturia in the lepromatous leprosy patients were significantly higher than in the controls ( $p<0.001, p<0.001 ; p=0.001, p<0.01 ; p=0.036, p<0.05$, respectively). No significant differences in the proteinuria, hematuria, sodium, or potassium levels were found between the leprosy and control groups $(p>0.05)$. On ultrasonographic examination, the prevalence of renal cortical cysts and renal cortical echogenicity in the leprosy patients was significantly higher than in the controls ( $p=0.020, p<0.05$, respectively). There were no significant differences in terms of nephrolithiasis, parapelvic cysts, or hydronephrosis between the leprosy and control groups $(p>0.05)$.

Conclusions: Evaluating the renal function in all leprosy patients is important to detect abnormalities and to prevent renal failure, which remains a potential cause of death in this disease.
\end{abstract}

Key words: leprosy, renal abnormalities, ultrasonography, Turkey.

\section{Introduction}

Leprosy is a chronic granulomatous infection caused by Mycobacterium leprae that primarily affects the peripheral nerve trunks and cutaneous nerves. Leprosy continues to be a worldwide challenge to health, with approximately 250,000 new cases being detected each year $[1,2]$. The disease is diagnosed on the basis of three criteria: characteristic skin lesions in association with thickened nerves, a demonstration of acid-fast bacilli in slit skin smears, and the histopathology of skin biopsies [3].

Renal abnormalities in leprosy have been previously described in the literature, although $M$. leprae does not ordinarily invade the renal parenchyma. However, most patients with renal involvement only have asymptomatic urinary abnormalities [4, 5]. Glomerulonephritis, nephrosclerosis, tubulointerstitial nephritis, amyloidosis, and granulomas are the most common renal pathologies found on biopsies or autopsies of leprosy patients [5-7]. Mitsuda and Ogawa first reported the presence of renal lesions in this disease via autopsy findings $[7,8]$. Renal involvement in leprosy may progress to end-stage renal disease and may, therefore, be an important cause of death in patients with leprosy [8]. In a review of 199 autopsies in a Brazilian study of leprosy patients, renal lesions were found to be present in the bodies of 144 (72\%) patients who presented with amyloidosis, glomerulonephritis, nephrosclerosis, tubulointerstitial nephritis, granulomas and other lesions [9]. Glomerulus injuries have also been described in histological findings. Proliferative mesangial glomerulonephritis is the most regularly described glomerular disease in leprosy; however, many other histological features have also been described [9-12]. The prevalence of glomerulonephritis has been

Address for correspondence: Savas Ozturk, Department of Dermatology, Medical Faculty, Balikesir University, 10145 Balikesir, Turkey, e-mail:drsozturk@gmail.com

Received: 13.11.2015, accepted: 22.05.2016. 
reported to range from $6 \%$ to $50 \%$ in leprosy patients [9]. Amyloidosis, with an incidence ranging from $2 \%$ to $55 \%$, is attributed to chronic granulomatous reactions caused by $M$. leprae and is manifested mainly by elevated proteinuria. Amyloidosis may then progress to chronic renal failure, one of the main causes of death in leprosy $[9,13$, 14]. The exact pathogenesis of renal lesions in leprosy is still unknown. Both immunoflourescent and electron microscopic studies suggest that the varied glomerular lesions found in these patients are immune complexmediated. Observers suggest that the reaction episode, multibacillary classification, and age are risk factors for renal function impairment in leprosy $[11,15,16]$.

\section{Aim}

The present study was undertaken to evaluate renal functional impairment in leprosy patients using clinical, biochemical, and ultrasonographic criteria.

\section{Material and methods}

This study was undertaken in the Department of Dermatology and Leprology at the Elazig Training and Research Hospital between September 2011 and June 2013. The local Ethics Committee gave approval for this research and written consent was obtained from all subjects. This study was conducted to detect renal involvement in patients with leprosy through the analysis of clinical, radiological, and laboratory data taken from both the patients' medical records and from current analyses. We enrolled 32 patients with lepromatous leprosy (8 females and 24 males) and 35 healthy controls (11 females and 24 males). Patients and control subjects were excluded if they had any co-morbidity, such as hypertension, diabetes, chronic urinary tract infection, or any other condition that could cause renal dysfunction. All of the leprosy patients had been diagnosed with lepromatous leprosy according to the classification proposed by the World Health Organization. They had also undergone the prescribed treatment, consisting of multidrug therapy. Patients who were enrolled in the study were questioned about their age, gender, age at onset, duration of disease, job, origin, address, telephone number, time of treatment, symptoms and signs, and laboratory findings (serum urea and creatinine, serum sodium $(\mathrm{Na})$ and potassium (K), and urinalysis). Proteinuria, hematuria, hemoglobinuria, and leukocyturia were analyzed with the use of reagent strips. We considered proteinuria and hemoglobinuria to be one or more "+" in the qualitative exam. Hematuria was considered to be one or more "+" in the qualitative exam and one or more erythrocytes per high-power field. Leukocyturia was defined as the presence of five or more leukocytes per high-power field. Renal failure was defined as a serum creatinine level $\geq 1.4 \mathrm{mg} / \mathrm{dl}$.

\section{Assessing renal involvement using transabdominal ultrasonography}

Ultrasonography (US) was performed using a SSA660A ultrasound system (Xario, Toshiba Medical Systems, Tochigi, Japan) and a 3.5-5-MHz convex probe. All US examinations were performed by a previously-designated and experienced radiologist (TO) who was blinded to all subjects. Renal longitudinal size, parenchymal thickness, cortical thickness, cortical echogenicity, and corticomedullary differentiation were all evaluated. Renal cortical echogenicity was compared with the echogenicity of the liver and renal medulla, and graded as follows: - grade 0: normal echogenicity lower than that of the liver, with maintained corticomedullary definition;

- grade 1: echogenicity the same as that of the liver, with maintained corticomedullary definition;

- grade 2: echogenicity greater than that of the liver, with maintained corticomedullary definition;

- grade 3: echogenicity greater than that of the liver, with poorly-maintained corticomedullary definition;

- grade 4: echogenicity greater than that of the liver, with a loss of corticomedullary definition.

\section{Statistical analysis}

Statistical analysis was performed using SPSS for Windows software (ver. 12.0; SPSS Inc., Chicago, IL, USA). Quantitative data were expressed as means \pm standard deviation (SD). The normality of data was analyzed using the Kolmogorov-Smirnov test. All numerical variables following a normal distribution were expressed as means \pm $\mathrm{SD}$, while data that did not follow a normal distribution were expressed as median values (interquartile range). Pearson's $\chi^{2}$ and Fisher's exact tests were used to compare the qualitative values. A Mann-Whitney $U$ test was used to compare the quantitative values of the two groups. In all analyses, $p<0.05$ was taken to indicate statistical significance.

\section{Results}

Of the 45 patients diagnosed with lepromatous leprosy who were followed in our outpatients clinic, 13 were excluded due to a co-morbidity (7 patients were hypertensive, 4 had type 2 diabetes mellitus, 1 had bladder cancer, and 1 had chronic renal failure). In total, $32 \mathrm{pa}-$ tients ( 24 males and 8 females; the leprosy group) and 35 healthy control subjects ( 24 males and 11 females; the control group) were included in this study. The mean age in the leprosy group was $66.3 \pm 9.2$ years, and the mean age in the control group was $66.0 \pm 8.5$ years. The clinical and epidemiological data of the patients and controls are summarized in Table 1. Age and gender were found to be similar between the groups.

Evaluation of the leprosy and control groups was carried out via an analysis of the clinical, radiological, and 
laboratory data obtained from both the patients' medical records and current examinations.

The mean urea and creatinine concentrations in the leprosy group $(62.3 \pm 42.2$ and $1.3 \pm 0.8$, respectively) were found to be significantly higher when compared with the controls ( $35.5 \pm 13.7$ and $0.9 \pm 0.3$, respectively) ( $p<$ $0.001, p<0.001 ; p=0.001, p<0.01$, respectively). Levels of creatinine above $1.4 \mathrm{mg} / \mathrm{dl}$ were detected in 6 (18.75\%) patients and levels of urea above $40 \mathrm{mg} / \mathrm{dl}$ were found in 20 (62.5\%). No significant differences were determined in the levels of $\mathrm{Na}$ and $\mathrm{K}$ between the leprosy and control groups $(p>0.05)$. From the urinalysis results, proteinuria was found in 9 (28.12\%) patients, hematuria in 16 (50.0\%), and leukocyturia in 16 (50.0\%). There were no significant differences in proteinuria or hematuria between the leprosy and control groups $(p>0.05)$. Leukocyturia was found to be significantly higher in the leprosy group (50.0\%) compared to the control group (26.0\%) ( $p=0.036, p<0.05$, respectively). The serum urea, creati-

Table 1. The clinical and epidemiological data of the patients and healty subjects

\begin{tabular}{lccc}
\hline Parameter & Patients & Controls & $P$-value \\
\hline Age [years] & $66.3 \pm 9.2$ & $66.0 \pm 8.5$ & 0.748 \\
\hline Gender, $n(\%)$ & Female: $8(25)$ & Female: $11(31)$ & 0.379 \\
& Male: $24(75)$ & Male: $24(69)$ & \\
\hline
\end{tabular}

nine, $\mathrm{Na}$ and $\mathrm{K}$ levels, and urinalysis results of the leprosy and control groups, are summarized in Table 2.

The results of the US examination show that the prevalence rates of renal cortical cysts and increased renal cortical echogenicity in the leprosy patients were significantly higher than in the controls $(p=0.020, p<0.05$, respectively). No significant differences were found in nephrolithiasis, parapelvic cysts, or hydronephrosis between the groups ( $p$ $>0.05$ ). Increased renal cortical echogenicity was observed in 13 patients, 3 of whom were classed as grade 2 while 10 were classed as grade 1 . Increased renal cortical echogenicity was observed in 5 controls, all of whom were classed as grade 1. The ultrasonographic results of the leprosy and control groups are summarized in Table 3.

\section{Discussion}

Leprosy is a multisystem infectious disease that can involve the kidneys in a variety of ways [11]. The exact pathogenesis of renal lesions in leprosy is still unclear [13]. Mycobacterium leprae does not appear to be directly involved in the creation of renal lesions [16], although it has been detected in the renal parenchyma of some patients, including the glomeruli $[9,11]$. Glomerular lesions are probably mediated by immunocomplexes $[12,13,16]$. Some studies have demonstrated that renal lesions are the main cause of death in leprosy patients [9, 13, 14, 16].

The results of this cross-sectional analysis of renal abnormalities in leprosy patients help to clarify the im-

Table 2. The serum urea, creatinine, $\mathrm{Na}$, $\mathrm{K}$ levels and urinanalysis reports of patients and controls

\begin{tabular}{|c|c|c|c|c|c|}
\hline \multirow[t]{2}{*}{ Parameter } & \multicolumn{2}{|c|}{ Lepromatous leprosy } & \multicolumn{2}{|c|}{ Controls } & \multirow[t]{2}{*}{$P$-value } \\
\hline & Mean & SD & Mean & SD & \\
\hline Urea [mg/dl] & 62.3 & 42.2 & 35.5 & 13.7 & $<0.001^{\star * *}$ \\
\hline Creatinine [mg/dl] & 1.3 & 0.8 & 0.9 & 0.3 & $0.001^{\star *}$ \\
\hline $\mathrm{Na}[\mathrm{mEq} / \mathrm{l}]$ & 140.5 & 3.1 & 139.3 & 3.4 & 0.163 \\
\hline $\mathrm{K}[\mathrm{mEq} / \mathrm{l}]$ & 4.4 & 0.6 & 4.4 & 0.5 & 0.620 \\
\hline Hematuria, $n$ (\%) & \multicolumn{2}{|c|}{$16(50)$} & \multicolumn{2}{|c|}{$16(46)$} & 0.458 \\
\hline Leukocyturia, $n$ (\%) & \multicolumn{2}{|c|}{$16(50)$} & \multicolumn{2}{|c|}{$9(26)$} & $0.036^{\star}$ \\
\hline Proteinuria, $n$ (\%) & \multicolumn{2}{|c|}{$9(28)$} & \multicolumn{2}{|c|}{$4(11)$} & 0.078 \\
\hline
\end{tabular}

${ }^{*} p<0.05,{ }^{* *} p<0.01,{ }^{* * *} p<0.001$.

Table 3. Ultrasonographic results of patients and controls

\begin{tabular}{|c|c|c|c|c|c|}
\hline \multirow[t]{2}{*}{ Parameter } & \multicolumn{2}{|c|}{ Patients } & \multicolumn{2}{|c|}{ Controls } & \multirow[t]{2}{*}{$P$-value } \\
\hline & $n$ & $\%$ & $n$ & $\%$ & \\
\hline Nephrolithiasis & 5 & 15.6 & 3 & 8.6 & 0.304 \\
\hline Cortical cyst & 18 & 56.2 & 10 & 28.6 & $0.020^{*}$ \\
\hline Parapelvic cyst & 1 & 3.1 & 0 & 0.0 & 0.478 \\
\hline Hydronephrosis & 3 & 9.4 & 2 & 5.7 & 0.457 \\
\hline Increased renal cortical echogenicity & 13 & 40.6 & 5 & 14.3 & $0.015^{*}$ \\
\hline
\end{tabular}

${ }^{*} p<0.05,{ }^{* *} p<0.01,{ }^{* * *} p<0.001$. 
portant aspects of renal involvement in leprosy. Some previous studies have found raised levels of serum urea in up to $53 \%$ of patients $[4,17]$, while others have failed to find any significant rise in serum urea levels even in reactional states $[4,5,18,19]$. In the present study, levels of creatinine above $1.4 \mathrm{mg} / \mathrm{dl}$ were detected in 6 (18.75\%) patients and levels of urea above $40 \mathrm{mg} / \mathrm{dl}$ were found in 20 (62.5\%). The mean urea and creatinine concentration levels of the leprosy patients were significantly higher compared to the controls. Singh et al. [20] found that $9.33 \%$ of leprosy patients exhibited increased serum creatinine levels. Of these, 5 patients had lepromatous leprosy. Oliveira et al. [16] reported that leprosy patients had a lower glomerular filtration rate compared to controls. In addition, the same research team observed a serum creatinine level of $>1.2 \mathrm{mg} / \mathrm{dl}$ in $8.5 \%$ of cases. Daher et al. [15] reported that the mean serum urea and creatinine levels of 923 leprosy patients were $30.5 \pm 24.7 \mathrm{mg} / \mathrm{dl}$ and $0.9 \pm 1.3 \mathrm{mg} / \mathrm{dl}$, respectively. The majority of these patients had been diagnosed with lepromatous leprosy. Renal dysfunction was identified in 35 (3.8\%) cases. da Silva Jr. and Daher [8] performed a retrospective analysis of 461 patients with leprosy in Brazil and detected levels of creatinine above $1.4 \mathrm{mg} / \mathrm{dl}$ in 40 (8.6\%) patients and levels of urea above $40 \mathrm{mg} / \mathrm{dl}$ in 52 (11.2\%). Most of these patients (45\%) had lepromatous leprosy, which suggests that this form of the disease has a stronger association with renal damage. They found the incidence of renal failure to be significantly higher among the lepromatous leprosy patients. In addition, the mean serum creatinine and urea levels were also significantly higher in the lepromatous leprosy group compared to both the control group and patients with tuberculoid leprosy. The authors of this study suggested that the high numbers of bacilli observed in lepromatous patients could be the cause of more frequent and prominent renal injuries. Nadeem et al. [4] observed abnormal serum urea levels in $6.66 \%$ of patients and abnormal serum creatinine levels in $16.66 \%$ of patients. Some studies found that multibacillary patients presented with a higher frequency of glomerular dysfunction [10, 13, 16, 21-25]. In our results, the serum urea and serum creatinine levels were higher compared to other studies because our patients had a greater mean age and all of them had been diagnosed with lepromatous leprosy.

The mean serum $\mathrm{Na}$ and $\mathrm{K}$ levels in our leprosy patients were within the normal range, and were also similar to the levels observed in other studies (mean values = $140.5 \pm 3.1 \mathrm{mEq} / \mathrm{l}$ and $4.4 \pm 0.6 \mathrm{mEq} / \mathrm{l}$, respectively).

Abnormalities on urinalysis have frequently been detected in leprosy patients and different studies have shown their prevalence to be extremely variable $[10,16$, 19]. In the present study, proteinuria was found in 9 patients (28.12\%), hematuria in 16 (50.0\%), and leukocyturia in 16 (50.0\%). Proteinuria and hematuria were higher in the lepromatous leprosy patients compared to the healthy controls. However, the difference was not found to be significant $(p<0.05)$. In a retrospective study conducted by de Silva Jr. and Daher [8], an analysis of 461 leprosy patients revealed that proteinuria was present in $36(7.8 \%)$ cases and hematuria in 60 (13.0\%). In the same study, mild proteinuria was observed in 6 (11.9\%) cases. In the present study, important urinalysis abnormalities were discovered, such as proteinuria, hematuria, hemoglobinuria, and leukocyturia. These abnormalities were more frequent in the lepromatous leprosy group. Oliveira et al. [16] reported the presence of hematuria in $27 \%$ of patients, a similar finding to other studies [8, 10, 13, 22]. Daher et al. [15] investigated 923 patients in Brazil and found proteinuria in $44(4.8 \%)$ cases, hematuria in 63 (6.8\%), and leukocyturia in 96 (10.04\%). In that study, high levels of proteinuria (50.9\% vs. $2.7 \%$ ) and hematuria ( $25.1 \%$ vs. 0\%) were found in the lepromatous leprosy patients compared with tuberculoid leprosy patients. Kirsztajn et al. [13] analyzed 96 patients and found proteinuria levels higher than $0.1 \mathrm{~g} / \mathrm{dl}$ in $2.1 \%$ of the cases. Shwe and Woodruff [26] analyzed 221 leprosy patients and identified a significantly higher frequency of proteinuria in the lepromatous leprosy patients, especially in those with a high bacterial index as well as those undergoing reaction episodes.

Singh et al. [20] found that 9 out of 75 study patients had hematuria. Proteinuria was observed in $10.66 \%$ of the cases. Of these, 6 (19.35\%) cases were classed as lepromatous leprosy.

Nadeem et al. [4] determined a low incidence of proteinuria and hematuria in leprosy patients and considered this to be related to the pattern of the disease. Kanwar et al. [17] identified a high incidence of proteinuria in their patients, and other studies have also found a high incidence of proteinuria $[15,17]$.

In our study, we found that the incidences of proteinuria, hematuria, and leukocyturia were higher compared to other studies since our patients had a greater mean age and all of them had been diagnosed with lepromatous leprosy.

Chronic kidney disease (CKD) is one of the most common causes of renal failure. The CKD can be diagnosed by its pathological abnormalities, changes in the levels of kidney function markers in the blood or urine, or by imaging investigations [27, 28]. US is the ideal imaging modality in CKD because of its non-invasiveness and because it provides easy accessibility and visualization of the kidneys. US is the first, and, in most cases, the only imaging investigation required in the work-up of chronic renal failure [27-30]. Previous studies have revealed that renal echogenicity and its grading correlates better with serum creatinine levels in CKD than with other sonographic parameters. As serum creatinine is an indicator of kidney function, renal echogenicity is a better parameter for estimating renal function, with the added advan- 
tage of irreversibility when compared to serum creatinine levels [27-32].

We found that renal cortical cysts and increased renal cortical echogenicity in lepromatous leprosy patients were significantly higher than in the control group ( $p=$ 0.020, $p<0.05$ ), but found no significant differences in nephrolithiasis, parapelvic cysts, or hydronephrosis between the leprosy and control groups $(p>0.05)$. Renal assessment by US in lepromatous leprosy has rarely been reported in the literature. Singh et al. [20] found that 7 out of 75 (9.45\%) patients (5 with lepromatous leprosy) exhibited some renal abnormality on US. Of these patients, 5 had a bilateral reduction in renal size and corticomedullary differentiation was lost in 3 patients. To the best of our knowledge, our study is the first to investigate the assessment by US of renal functional status in leprosy patients.

\section{Conclusions}

Leprosy can cause important renal abnormalities. Renal involvement in leprosy appears to be related to the quantity of bacilli present in the body. The evaluation of renal function in all leprosy patients is important to detect abnormalities and to prevent renal failure, which is still considered to be a potential cause of death in this disease. Leprosy patients should be monitored via urinalysis, creatinine levels, and US to detect renal involvement.

\section{Acknowledgments}

This study was presented at the XXV National Congress of Dermatology, 2014, Antalya, Turkey.

\section{Conflict of interest}

The authors declare no conflict of interest.

\section{References}

1. Rodrigues LC, Lockwood DNJ. Leprosy now: epidemiology, progress, challenges, and research gaps. Lancet Infect Dis 2011; 11: 464-70.

2. Kulkarni M, Chauhan V, Bharucha M, et al. MRI imaging of ulnar leprosy abscess. Assoc Physicians India 2009; 57: 175-6.

3. Grimaud J, Vallat JM. Neurological manifestations of leprosy. Rev Neurol 2003; 159: 979-95.

4. Nadeem M, Garg BR, Das AK. Renal functional status in leprosy. Indian J Dermatol Venereol Leprol 1995; 61: 133-6.

5. Bajaj AK, Gupta SL, Sinha SN, et al. Renal functional status in lepromatous leprosy. Int J Lepr 1981; 49: 37-41.

6. Mclver LJ, Parish ST, Jones SP, et al. Acute glomerulonephritis in a child with multidrug-resistant tuberculosis and multibacillary leprosy. Med J Aust 2011; 195: 150-2.

7. Chugh KS, Damle PB, Kaur S, et al. Renal lesions in leprosy amongst north Indian patients. Postgrad Med J 1983; 59: 707-11.
8. da Silva Júnior GB, Daher Ede F. Renal involvement in leprosy: retrospective analysis of 461 cases in Brazil. Braz J Infect Dis 2006; 10: 107-12.

9. Nakayama EE, Ura S, Fleury RN, Soares V. Renal lesions in leprosy: a retrospective study of 199 autopsies. Am J Kidney Dis 2001; 38: 26-30.

10. Nigam P, Pant KC, Kapoor KK, et al. Histo-functional status of kidney in leprosy. Indian J Lepr 1986; 58: 567-75.

11. Ahsan N, Wheeler DE, Palmer BF. Leprosy-associated renal disease: case report and review of the literature. I Am Soc Nephrol 1995; 5: 1546-52.

12. Weiner ID, Northcutt AD. Leprosy and glomerulonephritis: case report and review of literature. Am J Kidney Dis 1989; 13: 424-9.

13. Kirsztajn GM, Nishida SK, Silva MS, et al. Renal abnormalities in leprosy. Nephron 1993; 65: 381-4.

14. Chugh KS, Sakhuja V. Renal lesions in leprosy. J Assoc Physicians India 1991; 39: 163-4

15. Daher EF, Silva GB Jr, Cezar LC, et al. Renal dysfunction in leprosy: a historical cohort of 923 patients in Brazil. Trop Doct 2011; 41: 148-50.

16. Oliveira RA, Silva GB Jr, Souza CJ, et al Evaluation of renal function in leprosy: a study of 59 consecutive patients. Nephrol Dial Transplant 2008; 23: 256-62.

17. Kanwar AJ, Baharija SC, Belhaj MS. Renal function status in leprosy. Ind J Lepr 1984; 56: 595-9.

18. Thomas G, Karat ABA, Rao PS, et al. Changes in renal function during reactive phases of lepromatous leprosy. Int J Lepr 1970; 38: 170-6.

19. Mittal MM, Agarwal SC, Maheshwari HB, et al. Renal lesions in leprosy. Arch Pathol 1972; 93: 8-12.

20. Singh S, Nigam PK, Sethi IM. Renal function status in leprosy. Indian J Dermatol Venereol Leprol 1997; 63: 162-5.

21. Gutman RA, Lu WH, Drutz DJ. Renal manifestations of leprosy: impaired acidification and concentration of urine in patients with leprosy. Am J Trop Med Hyg 1973; 22 (Suppl. 2): 223-8.

22. Ponce $P$, Ramos A, Ferreira ML, et al. Renal involvement in leprosy. Nephrol Dial Transplant 1989; 4 (Suppl. 2): 81-4.

23. Phadnis MD, Metha MC, Bharaswadker MS, et al. Study of renal changes in leprosy. Int J Lepr Other Mycobact Dis 1982; 50 (Suppl. 2): 143-7.

24. Kaur S. Renal manifestation of leprosy. Indian J Lepr 1990; 62 (Suppl. 3): 273-80

25. Madiwale CV, Mittal BV, Dixit M, Acharya VN. Acute renal failure due to crescentic glomerulonephritis complicating leprosy. Nephrol Dial Transplant 1994; 9 (Suppl. 2): 178-9.

26. Shwe T, Woodruff AW. Renal involvement in leprosy. Trans R Soc Trop Med Hyg 1972; 66: 26-7.

27. Siddappa JK, Singla S, Al Ameen M, et al. Correlation of ultrasonographic parameters with serum creatinine in chronic kidney disease. J Clin Imaging Sci 2013; 3: 28.

28. O'Neill WC. Sonographic evaluation of renal failure. Am J Kidney Dis 2000; 35: 1021-38.

29. Moghazi S, Jones E, Schroepple J, et al. Correlation of renal histopathology with sonographic findings. Kidney Int 2005; 67: 1515-20.

30. Hricak H, Cruz C, Romanski R, et al. Renal parenchymal disease: sonographic-histologic correlation. Radiology 1982; 144: 141-7.

31. Päivänsalo M, Huttunen K, Suramo I. Ultrasonographic findings in renal parenchymal diseases. Scand J Urol Nephrol 1985; 19: 119-23.

32. Perrone RD, Madias NE, Levey AS. Serum creatinine as an index of renal function: new insights into old concepts. Clin Chem 1992; 38: 1933-53. 